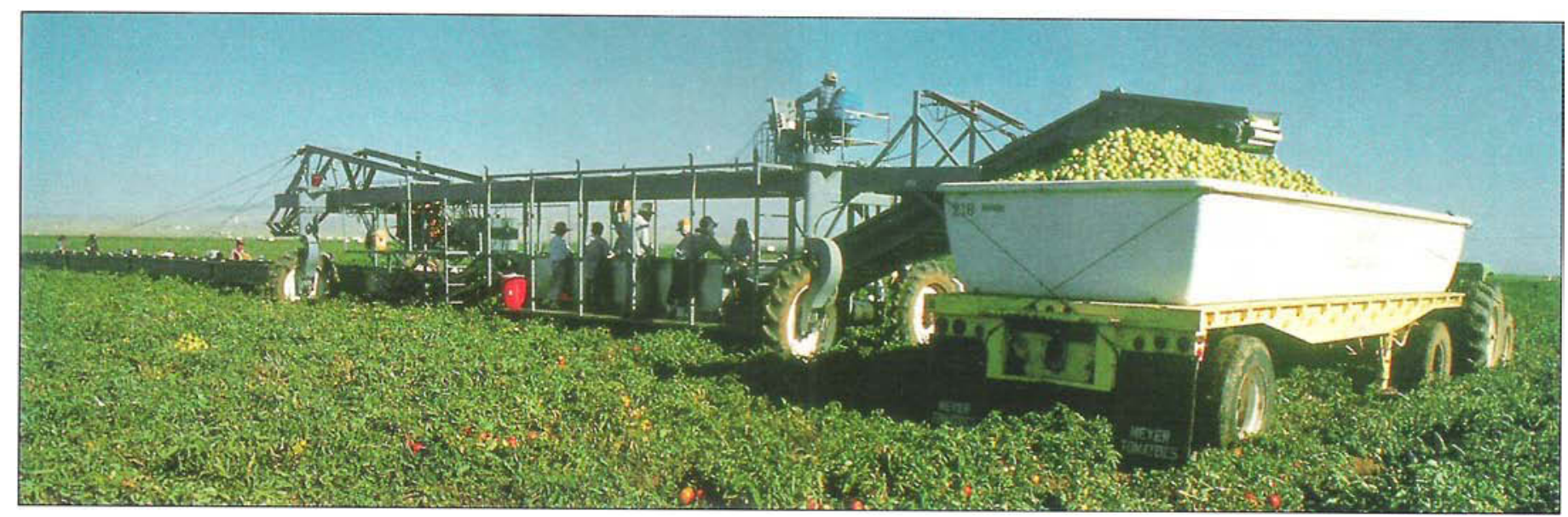

"Mechanical harvesting" doesn't say it all - a dozen or more laborers must work together to operate this tomato harvester, filling tractor trailers with green fruit destined to ripen fully

\title{
Bush tomatoes show very low levels of pesticide residues
}

\author{
Frank V. Sances \\ Nick C. Toscano
}

\begin{abstract}
Do pesticide residues persist on bush tomatoes? Apparently not or at least not much, according to a new study. When fruit was treated directly, then washed and brushed during normal postharvest handling, most - if not all - chemical residues were reduced by 50 to 95\%. Extensive sampling at commercial packing facilities showed no detectable residues.
\end{abstract}

One response to demands by legislators, special interest groups, major industry retailers, and consumers for "residue-free" produce from growers has come in the form of basic studies to provide California tomato growers with new information on how to minimize pesticide residues. The studies took place in two simulated field usage programs and three actual commercial fields in California. Five commonly used pesticides were studied in simulated usage conditions and three others in commercial plantings. The focus study materials were: chlorothalonil, dimethoate, fenvalerate, methamidophos, and methomyl.

Meanwhile, the U.S. Food and Drug Administration (FDA) and Envitonmental Protection Agency (EPA) are re-evaluating registrations for numerous commercial pesticides, including chlorothalonil, meth-

\author{
- Lyle K. Gaston
}

omyl, and several pyrethroid insecticides. At present, relatively high tolerances exist for these "first-line-of-defense" materials. However, the FDA may at any time reevaluate the materials and require lower market basket residues. Certainly, marketplace surveillance testing of produce for pesticide residues by government and industry is likely in the near future.

\section{Simulated field usage}

The simulated field usage programs were conducted on field-grown bush tomatoes in California's south coastal area. Freshmarket bush tomatoes (Jackpot Cultivar) were planted in 1,000-square-foot field plots at 5,500 plants per acre from May 29 through August 27, 1990. Two experimental treatment programs, which were replicated four times in a standard randomized complete block design, were established:

1. MUP, or Maximum Usage Program, where maximum legal rates of the five study pesticides were applied frequently to simulate a maximum residue level.

2. SUP, or Scheduled Usage Program, where the same materials were applied at maximum rates, but half as often, to approximate a commercial pest control program used during high pest-pressures periods.

To provide chemical-free tomatoes for baseline study purposes, field plots of fruit that were treated only with nonsynthetic materials such as Bacillus thuringiensis (Bt) and botanical insecticides were also established.

All label restrictions, including required intervals between application and harvest, were observed for the synthetic materials. The treatments were applied by tractor-mounted spray equipment at 150 pounds per square inch (psi), using multiple-nozzle booms delivering from 50 to 130 gallons per acre to maximize the amount of material contacting developing fruit.

To compare residues on field-gate and packed tomatoes from the two simulated usage programs, samples were taken from the respective treatment plots and split into two groups. One group contained unwashed, field-gate fruit; the other contained fruit that was washed for 5 minutes in room-temperature, $\mathrm{pH}$-neutral water. The latter group simulated basic postharvest commercial washing at the UC Riverside laboratory, and constituted the "packed" group. Residue analysis was then performed on whole fruit using FDAapproved methods from each replicated MUP, SUP, and control treatment.

Residues of chlorothalonil and methamidophos were present at harvest gate; no residues of the other studied pesticides were found. Chlorothalonil, found on unwashed field fruit at 0.13 part per million (ppm), was eliminated after simulated postharvest handling. 
Methamidophos, the most persistent material used, was detectable only in the MUP program at $0.15 \mathrm{ppm}$ on unwashed fruit and 0.17 ppm on washed fruit. It should be noted that methamidophos was used excessively in the MUP program. Even then, the resulting residue was 15 to $17 \%$ of the EPA acceptable tolerance of 1 $\mathrm{ppm}$. These data indicate that unwashed, field-gate tomatoes have less pesticide residues than was originally assumed. It also shows that even the excessive highvolume ground applications made during the MUP program did not create fruit residues that were above tolerance levels. In most cases, residues were undetectable.

These data agree with FDA residue data for a 5-year period that identified methamidophos as the pesticide most often found as a residue on tomatoes. These findings also show that postharvest washing had no affect on removing methamidophos residues.

\section{Actual field usage}

Three representative California growing locations were selected for the actual field usage programs. Desert, coastal, and Central Valley production districts were the Brawley, King City, and Huron areas, respectively. These sites were chosen for their climatic conditions, varying pest pressures, and subsequent pesticide usage patterns.

At Brawley and Huron, cooperating growers followed their typical pesticide usage programs. All fields were managed using commercial IPM methods, with field pest control advisors using pesticides on an as-needed basis. All pesticides were applied less frequently than even the SUP treatments in the simulated field usage programs. Complete records were maintained from three study fields of 10- to 25 acre plantings each (table 1). Besides the five focus materials, growers also applied diazinon, dicofol, and triadimefon in these

TABLE 1. Pesticides used in tractor-applied simulated usage programs on experimental tomato plantings and actual commercial IPM-grown plantings, with frequency and days to harvest for applications (South Coast Research and Extension Center, Irvine, for experimental plantings; Brawley, King City, and Huron, California, for commercially managed plantings)

\begin{tabular}{|c|c|c|c|c|c|c|c|}
\hline \multirow[b]{3}{*}{ Common name } & \multirow[b]{3}{*}{ Formulation } & \multirow[b]{3}{*}{ Rate } & \multicolumn{4}{|c|}{ Days to harvest } & \\
\hline & & & \multicolumn{2}{|c|}{$\begin{array}{l}\text { Experimental } \\
\text { simulations }\end{array}$} & \multicolumn{3}{|c|}{$\begin{array}{c}\text { Commercial } \\
\text { plantings }\end{array}$} \\
\hline & & & MUP* & SUPt & Brawley & King City & Huron \\
\hline Chlorothalonil & $500 S$ & $\begin{array}{l}\mathrm{Ib} / \mathrm{ai} / \mathrm{ac} \\
1.6-2.1\end{array}$ & $\begin{array}{c}61,46 \\
35,21,7\end{array}$ & 35,7 & $-\S$ & 3 & - \\
\hline Diazinon & AG500 & 0.5 & - & - & - & - & 90 \\
\hline Dicofol & MFL & 0.75 & - & - & - & - & 60 \\
\hline Dimethoate‡ & 2.67EC & 0.5 & $\begin{array}{c}35,26 \\
21,14,7\end{array}$ & $35,26,7$ & - & 25,8 & $\begin{array}{c}106,103 \\
97,90\end{array}$ \\
\hline Fenvalerate $\ddagger$ & $0.66 \mathrm{EC}$ & 0.05 & $\begin{array}{l}35,26 \\
21,14,7\end{array}$ & $35,26,7$ & $\begin{array}{c}46,33 \\
19\end{array}$ & 3 & 48,28 \\
\hline Methamidophosł & $4.0 \mathrm{~S}$ & 1.0 & $\begin{array}{c}61,46 \\
35,21,7\end{array}$ & 35,7 & $\begin{array}{l}89,66 \\
57,33\end{array}$ & 25,8 & 48 \\
\hline Methomyl & $1.8 \mathrm{~L}$ & 0.9 & $\begin{array}{c}35,26 \\
21,14,7\end{array}$ & $35,26,7$ & - & 25,3 & $\begin{array}{c}103,97,81 \\
71,48,28\end{array}$ \\
\hline Triadimefon & 50WP & $0.06-0.13$ & - & - & - & - & $\begin{array}{l}81,71 \\
48,28\end{array}$ \\
\hline
\end{tabular}

"MUP is the maximum usage program.

†SUP is the scheduled usage program.

‡Focus study materials in simulated studies and washing experiments.

$\S-=$ Pesticide not applied during growing season.

TABLE 2. Surfactant efficiency in residue removal from tomatoes with laboratory washing equipment, UC Riverside

Percentage removal by pesticide*

\begin{tabular}{lllcccc} 
Surfactant & Type & Chlorothalonil & Dimethoate & Methamidophos & Methomyl & Fenvalerate \\
\cline { 5 - 7 } Triton $\mathrm{B} 1956$ & Nonionic & $85 \mathrm{~b}$ & $10 \mathrm{a}$ & $18 \mathrm{a}$ & $33 \mathrm{a}$ & $26 \mathrm{~b}$ \\
Tween 20 & Nonionic & $94 \mathrm{ab}$ & $30 \mathrm{a}$ & $40 \mathrm{a}$ & $55 \mathrm{a}$ & $56 \mathrm{a}$ \\
NaLauSO & Anionic & $96 \mathrm{a}$ & $25 \mathrm{a}$ & $35 \mathrm{a}$ & $50 \mathrm{a}$ & $52 \mathrm{a}$ \\
Water only & & $89 \mathrm{ab}$ & $17 \mathrm{a}$ & $25 \mathrm{a}$ & $47 \mathrm{a}$ & $27 \mathrm{~b}$ \\
\hline
\end{tabular}

*Numbers followed by same letter not significantly different. DMRT, .P $\geq 0.01$

acres, and our tests also checked the fruit for these chemicals.

Because of the extremely low incidence of harvest residues found at these locations, the cooperating grower at King City was asked to apply the five study pesticides just before harvest. The pesticides were air-applied at their respective maximum rates and minimum preharvest intervals. These treatments were in addition to the normal products applied during crop development. Resulting residues, which were obtained using FDA-approved methods, are shown in figure 1. All pesticides used during the study are shown as detectable or nondetectable at a detection limit of $0.05 \mathrm{ppm}$. Throughout these extensive samplings, no pesticide residues were detected on fruit sampled at field-gate or following postharvest washing and handling.

\section{Simulated washing}

Four factors that may influence pesticide removal during postharvest processing are wash water temperature and $\mathrm{pH}$, detergent rinsing, and physical brushing. To evaluate these treatments' effectiveness for residue removal, researchers needed tomatoes with sufficient residues. Fruit from the actual and simulated field studies, however, lacked such residues. To obtain fruit for the washing studies, the researchers established a separate field plot near King City in August 1990. All foliage was trimmed from plants in this plot to expose the fruit completely 4 days before it was to be harvested. The fruit was then treated directly with the five study pesticides at 100 gallons per acre, $40 \mathrm{psi}$, and five times their legal usage rates. Tomatoes were left attached to plants for 48 hours to enhance absorption through the skin and to allow sufficient time for solvents to evaporate. The fruit was then harvested and taken to the UC Riverside laboratory.

Before the candidate fruit were washed, all samples were tested to determine the amount of residues present. Residue levels of more than 3 ppm were noted for all materials except fenvalerate, which had prewash residues of $0.54 \mathrm{ppm}$. Using controlled washing treatments, groups of 25 fruit, replicated four times, were gently shaken in various types of aqueous solutions for 5 minutes to simulate the maximum wetted state of commercially handled fruit in the packing house. The fruit were then immediately analyzed using sample compositing, statistical subsampling, extraction, and gas chromatography analysis (per FDA guidelines).

Results of these tests follow below.

Water temperature. Many California tomato packers heat primary wash tank water to maintain fruit quality by preventing water absorption, which occurs when 


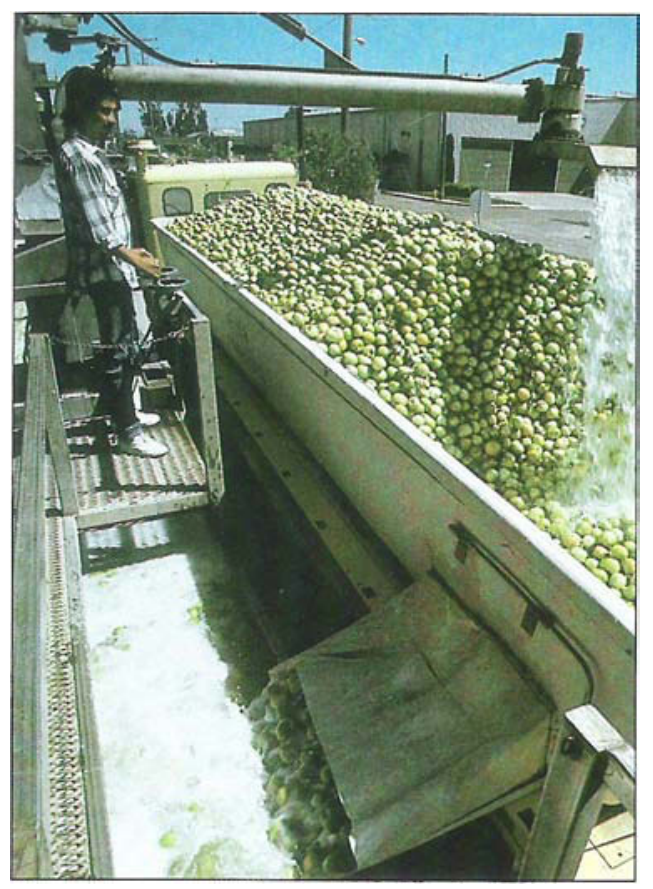

fruit pulp is warmer than the surrounding wash water. The required water temperature is usually $10^{\circ} \mathrm{F}$ higher than the fruit. Figure 2 presents data for fruit rinsed for 5 minutes in neutral water at $70^{\circ}$ and $100^{\circ} \mathrm{F}$.

Among the pesticides studied, fenvalerate benefited most from the hot water treatment: $38 \%$ more residue was removed than when the fruit was washed with room-temperature water. Other materials showed improvements of 13 to $17 \%$; however, this trend was statistically significant only for fenvalerate.

Water $\mathbf{p H}$. Room-temperature rinse waters at $\mathrm{pH} 4,7$, and 10 were compared for their effect on pesticide residue removal. A range of $\mathrm{pH} 4$ to $\mathrm{pH} 10$ was used because $\mathrm{pH}$ extremes outside this range may affect the shelf life of fruit. Also, high $\mathrm{pH}$ levels are not economically feasible for commercial use. Results from these analyses by $\mathrm{pH}$ concentration are shown in figure 3 . In general, most $\mathrm{pH}$ extremes were not significantly better than normal $\mathrm{pH} 7$ water, which reduced fruit residues between 20 and $95 \%$ depending on the pesticide present.

Detergent rinsing. Various commercial products that contain detergents and soap are available for washing residues from fruit. Of these, the anionic or nonionic type detergents are considered potentially beneficial when used singly or in combinations. Table 2 shows comparisons between surfactant types where two nonionic common detergents, Triton B1956 and Tween 20, were used at $0.1 \%$ solution strength. The anionic surfactant used was sodium laurel sulfate $\left(\mathrm{NaLauSO}_{4}\right)$, also at $0.1 \%$ concentration.

These data demonstrate two trends in the use of detergents to remove pesticide residues:

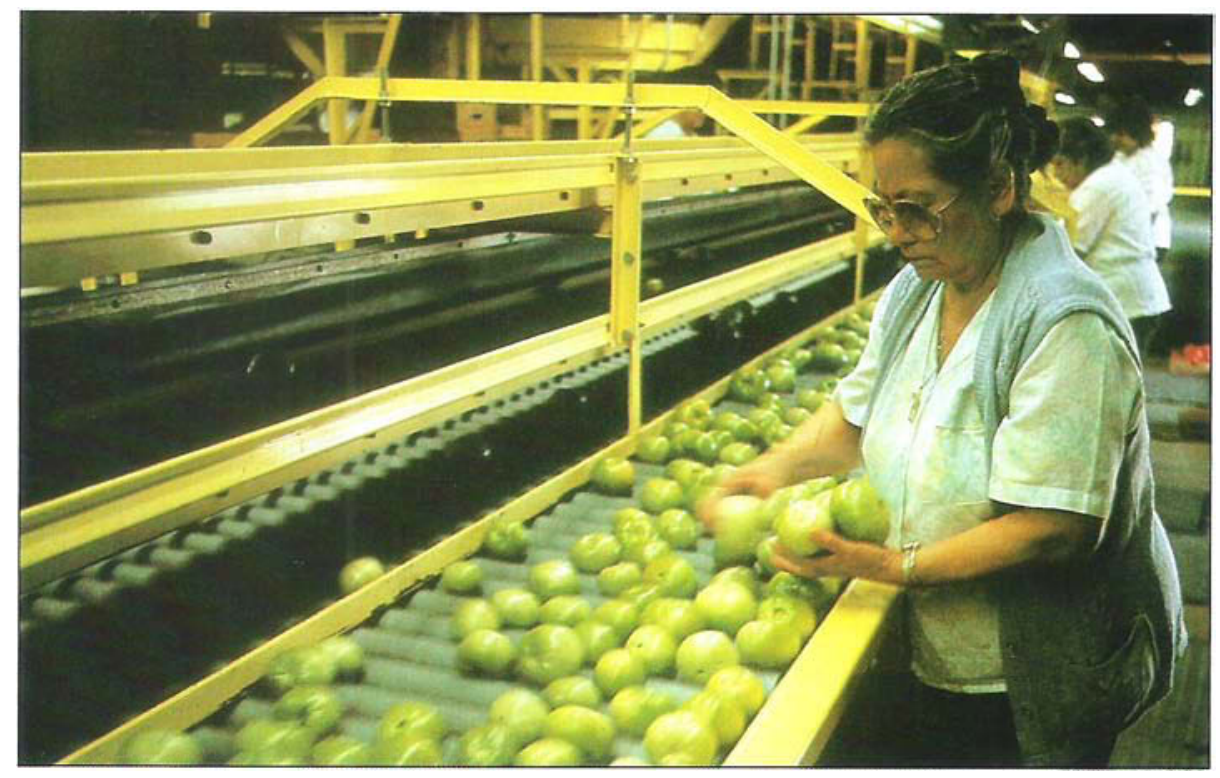

Trailer bins full of fresh-picked tomatoes (left) are flooded with water that will wash away pesticide residues and transport the fruit into the packing facility. After having been mechanically brushed, dipped, sprayed, and dried, fruit were sampled (right) at the end of the packing line.

FIELD GATE
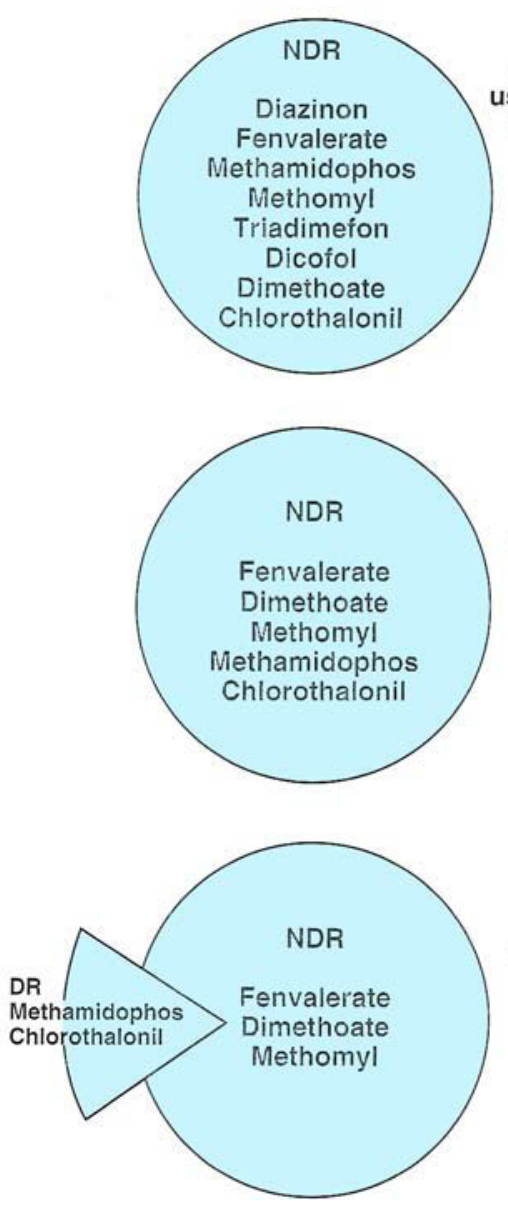

DR - Dectable residues

NDR - No dedectable residues (detection limit $=0.05$ ppm)

Fig. 1. Two pesticide usage programs (top and middle) out of three showed no detectable residues (NDR) on field-gate and packed tomatoes. For the third (bottom) program, growers applied maximum allowable levels of five pesticides as shortly before harvest as legally allowed.

\section{PACKED}
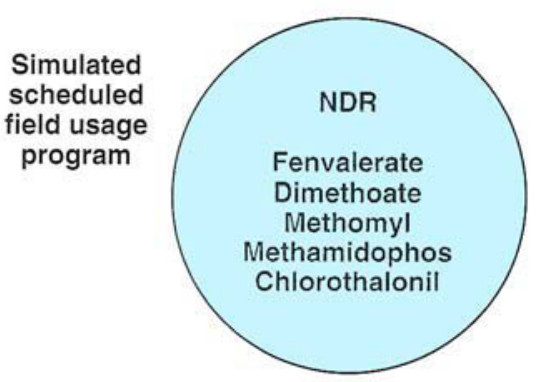

Simulated maximum field usage program

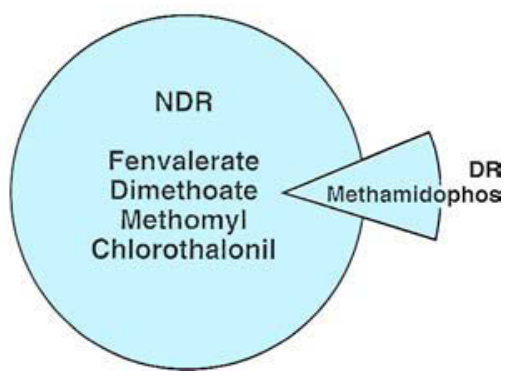




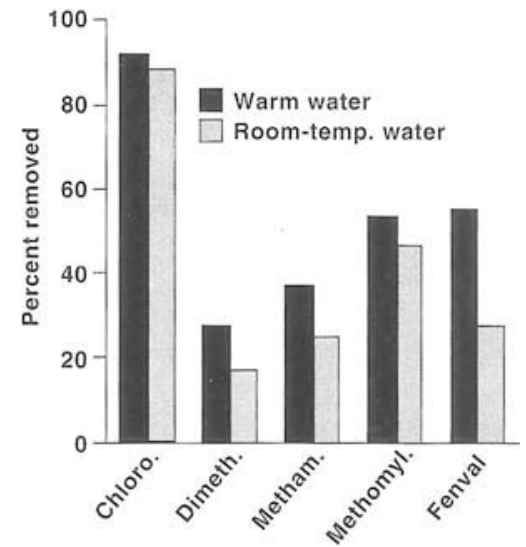

Fig. 2. Comparison of pesticide residue removal from field tomatoes using warm and room-temperature water rinses. Only fenvalerate showed significant change with warm water.

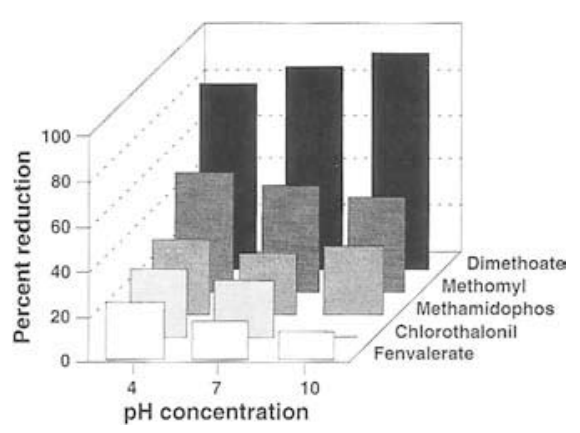

Fig. 3. In removing pesticide residues from field tomatoes, data show little effect, under experimental conditions, from using high, medium, or low-pH rinse water. Percentage removal was calculated from replicated samples of fruit from unrinsed (dry) and water-rinsed (pH 4, 7, 10) treatments.

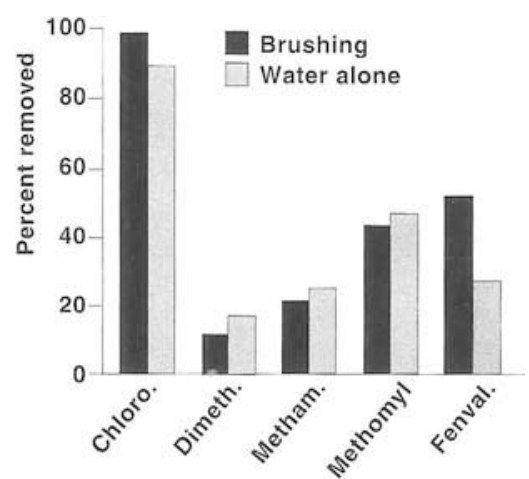

Fig. 4. Investigators compared residue removal when wet fruit were either brushed with polystyrene packing-line rollers or simply rinsed with water for the same amount of time. Again, only fenvalerate removal was significantly different.

1. The amount of residue removed varies dramatically, depending on the pesticide used. Chlorothalonil and methomyl residue levels, for example, did not significantly drop with detergent washing as compared with water-only washing. The systemic materials dimethoate and methamidophos had lower overall resi- dues with detergent washing, but also not significantly lower. In the case of fenvalerate, however, residue removal was doubled after rinsing with either Tween 20 or $\mathrm{NaLauSO}_{4}(\mathrm{P} \geq 0.01)$.

2. These data show a marked difference depending on which detergent was used. Although both Triton B1956 and Tween 20 are nonionic detergents, Triton B1956 was essentially no more effective than water alone. Conversely, Tween 20 tended to improve residue removal across all materials tested, particularly with fenvalerate, where residues were reduced by $56 \%$ following washing. Sodium laurel sulfate also performed well, removing $52 \%$ of the fenvalerate as compared with water only, which removed $27 \%$.

Brushing. Packing house brushing is commonly used to remove debris from field-grown fruit and create an aesthetically pleasing "luster" on tomatoes. This process was simulated in the laboratory by constructing a mechanical roller-brushing device using polystyrene in-line foam rollers rotating at 100 revolutions per minute $(\mathrm{rpm})$. Tomatoes were wetted with roomtemperature water at $\mathrm{pH} 7$ and brushed for 5 minutes. Results of analysis from brushed and unbrushed fruit are shown in figure 4.

These data are similar to other treatments where maximum benefit of brushing was achieved in removing fenvalerate. Residue removal of this pesticide by brushing was double that obtained when only water washing was used to remove it. Brushing also removed significantly more chlorothalonil ( $P \geq 0.01)$. Conversely, the systemic and semisystemic materials methamidophos, dimethoate and methomyl were unaffected by brushing. These were probably adsorbed into the fruit cuticle and therefore not as easily separated from fruit surfaces. Generally, this practice is probably efficient in removing residue only with macromolecules, such as fenvalerate, which is not adsorbed into the fruit cuticle and can be more easily separated from the surface of the fruit.

An additional treatment that may improve brushing efficiency is the adding of surfactants in solution to the brushing apparatus. This may optimize residue removal of persistent nonsystemic materials, such as fenvalerate, during postharvest handling.

\section{Conclusions}

These studies indicate that the occurrence and magnitude of persistent pesticide residues on bush tomatoes are minor. The use of FDA laboratory analysis procedures and extensive sampling of numerous samples taken from commercial packing facilities resulted in no detectable residues being found, with one exception. This was in simulated tractor-applied field plots with methamidophos. When used excessively, it persisted through normal postharvest processing.

The tomato production system appears at present to be well suited for minimizing pesticide residues on the finished product. Using commercial IPM practices, current field usage involves applying growing season pesticides much less frequently than did programs of the past. In field and laboratory, it was observed that applications made early in the tomato growing season, when plant canopies have not developed yet, result in the greatest contact between pesticide spray and fruit. Conversely, when canopies are well developed later in the season, fruit exposure, and thus pesticide fruit contact, is minimal.

Most early season residues on fruit naturally degrade with weathering and plant metabolism. Residues are also diluted as fruit grow larger during maturation. Near harvest, aircraft-applied low-dilution treatments are mainly limited to the nonharvestable portion of the crop. This is particularly true for bush tomatoes, which develop extensive plant canopies by harvest time.

The overall conclusion of this research is that present postharvest handling practices using room-temperature water, coupled with standard packing line brushing, are already near optimum in removing surface residues. The only addition to current practices would be to add an effective surfactant, such as Tween 20 or $\mathrm{NaLauSO}_{4}$, to the wash water and to use warm water where available if persistent pesticides must be used in the field near harvest. These, as well as other factors previously discussed, contribute to a very low incidence of pesticide residues on commercially produced bush tomatoes in California.

Further, from the empirical data presented here, growers are advised to avoid using highly systemic materials near harvest to minimize residues, since washing only minimally affects such materials. Proper residue management of tomatoes, therefore, involves both field and postharvest practices where appropriate nonpersistent pesticides are used during critical preharvest periods, and water and fruit brushing are used during postharvest handling.

F. V. Sances is a Postgraduate Research Assistant at UC Riverside; N. C. Toscano is Associate Dean, Division of Agriculture and Natural Resources, UC Riverside; and L. K. Gaston is a chemist in the Department of Entomology, UC Riverside.

The authors gratefully acknowledge the financial support of the Fresh Market Tomato Advisory Board and the Western Region Pesticide Impact Assessment Program. 